\title{
MUSIC RECOMMENDATION SYSTEM BASED ON FACIAL EMOTION RECOGNITION
}

Deny John Samuvel

Department of Electronics and Communication Engineering, Kalasalingam Academy of Research and Education,

Krishnankoil, Virudhunagar Dt., (India). E-mail: deny.j@klu.ac.in ORCID: http://orcid.org/0000-0001-6515-3575

B. Perumal

Department of Electronics and Communication Engineering,

Kalasalingam Academy of Research and Education, Krishnankoil, Virudhunagar Dt., (India). E-mail: palanimet@gmail.com ORCID: https://orcid.org/0000-0003-4408-9396

Muthukumaran Elangovan

Department of Electronics and Communication Engineering,

Dr. B. R. Ambedkar Institute of Technology, Pahargaon, Port Blair, (India). E-mail: reachmkumaran@gmail.com ORCID: https://orcid.org/0000-0002-0763-9902

\section{Citación sugerida:}

Samuvel, D. J., Perumal, B., y Elangovan, M. (2020). Music recommendation system based on facial emotion recognition. 3C Tecnología. Glosas de innovación aplicadas a la pyme. Edición Especial, Marzo 2020, 261-271. http://doi.org/10.17993/3ctecno.2020.specialissue4.261-271

\section{Suggested citation:}

Samuvel, D. J., Perumal, B., \& Elangovan, M. (2020). Music recommendation system based on facial emotion recognition. 3C Tecnología. Glosas de innovación aplicadas a la pyme. Edición Especial, Marzo 2020 , 261-271. http://doi.org/10.17993/3ctecno.2020.specialissue4.261-271 


\section{ABSTRACT}

Face recognition technology has widely attractedattention due to its enormous application value and marketpotential. It is being implemented in various fields like securitysystem, digital video processing, and many such technologicaladvances. Additionally, music is the form of art, which isknown to have a greater connection with a person's emotion. Ithas got a unique ability to lift up one's mood. Relatively, thispaper focuses on building an efficient music recommendationsystem which determines the emotion of user using FacialRecognition techniques. The algorithm implemented wouldprove to be more proficient than the existing systems. Moreover, on a larger dimension, this would render salvage oftime and labor invested in performing the process manually. The overall concept of the system is to recognize facial emotionand recommend songs efficiently. The proposed system will beboth time and cost efficient.

\section{KEYWORDS}

Recognition, Artificial intelligence, OpenCV Application. 


\section{INTRODUCTION}

Artificial intelligence, an extensive, prominent andimperative domain that has attracted a lot of researchers andprograms in recent times. This particular domain has takenover the world in very short notice. It is incorporated in over daily life in the form of chatbots, digital assistants like Siriand several other technology-based systems. One of the most prominent powers up of artificialintelligence is face recognition techniques. The basicexample of its usage is the grouping of Google Photos of aparticular person.

There are many existing systems that could recognize facialemotions. On the other hand, there are systems thatrecommend music. Bringing together, a system which willrecommend music by recognizing the mood of the user fromfacial emotions is the overall concept described in the paper. Emotion recognition would have larger scope in the nearfuture in fields like robotics for efficient sentimentalanalysis without the involvement of another human.

\section{RELATED WORK}

A few methodologies have been proposed and embraced to group human feelings successfully. Most of themethodologies laid their emphasis on seven essential feelings which are steady over age culture or different characters.

Describes the advantages of using OpenCV, especially the Adaboost algorithm, in the process of face recognition. Detecting and recognition of face in complicated color images can be achieved using a combination of a particular algorithm with AdaBoost algorithm. It also talks about the disadvantages of using a timer in face detection.

Proposes on utilizing Support Vector Machines (SVM) as the primary characterization technique to order eight facial feelings. The faces distinguished utilizing channels in OpenCV and changed over to Greyscale. The paper likewise explains on robotized constant coding of outward appearances in nonstop video gushing, which is feasible forapplications in which frontal perspectives can be accepted utilizing webcam.

The creator proposed a calculation to produce a subset ofa unique playlist or a custom playlist related to the feeling perceived. The picture to be prepared was acquired from 
aweb camera or the hard circle itself. The picture is expose to improvements, where a few mapping and upgrade procedures are connected to reestablish required differentiation of the picture. Preparing and arrangement are maintained by "one versus all" approach of SVM to encourage multi-class characterization.

Proposes on the utilization of profound convolutional neural networks. It depends on solid face acknowledgment convolutional systems, which can be effectively tweaked toplay out the feeling acknowledgment task. Visual models are supplemented with sound highlights for better face acknowledgment.

Aids in the music suggestion framework which is additionally a significant module of the proposed framework. It discusses highlights to be removed from the music to characterize its mind-set.

The paper depicts utilizing Thayer's model of mind-sets toperceive the state of mind of the music piece. The edge level of a music piece is resolved and the feeling it brings is perceived via prepared neural systems.

\section{METHODOLGY}

Compared to other algorithms used in previous systems, the proposed algorithm is proficient enough to battle large pose variations. Large pose variations tend to disrupt the efficiency of pre-existing algorithms. To reduce this Standard image input format is taken. Few systems detect the faces first and then locate them. On the other hand, rarely, some other algorithms detect and locate the faces at the same time. Every face detection algorithm usually has common steps. First, to achieve a response time, then to perform data dimension. Focusing on data dimension a few algorithms extract facial measurements and the next react certain relevant facial region. Advantages of the proposed algorithm Using the static image gives a great advantage on the defect of pose variations. The three most faced problems are the presence of unidentified elements like glasses or beard, quality of static images and unidentifiable facial gesture. Face Feature Extraction Pictures are spoken to as weight edeigen vectors that are consolidated and known as "Eigenfaces". One of the focal points taken by Eigen faces is the comparability between the pixels among pictures by methods for their covariance network. 
Following are the means required to perceive the outwardappearances utilizing this Eigenfaces approach:

Let $X=\left\{x_{1}, x_{2}, \ldots, x_{n}\right\} x_{i} \in R^{d}$

Here $X$ be a random vector with observations.

1. Calculate the mean $\mu$ :

$$
\mu=\frac{n}{1} \sum_{i=1}^{n} x_{i}
$$

2. Calculate the covariance matrix $S$ :

$$
S=\frac{n}{1} \sum_{i=1}^{n}\left(x_{i}-\mu\right)\left(x_{i}-\mu\right)^{T}
$$

3. Compute the eigenvectors $v_{i}$ and eigenvalues $\lambda_{\mathrm{i}}$ of $S$ :

$$
S v_{i}=\lambda_{i} v_{i}, i=1,2, \ldots, n
$$

4. The eigenvectors are arranged by their egeinvalue in descending order:

$$
y=W^{T}(x-\mu)
$$

5. Calculate eigenfaces.

Eigen Faces: Not all the parts of the face are important for emotion recognition. This key fact is considered to be important anduseful. Face recognition techniques focus on recognizingeyes, nose, cheek and forehead and how the change with respect to each other. Overall, the areas with maximumchanges, mathematically, areas with high variations are targeted. When multiple faces are considered, they are compared by detecting these parts of the faces becausethese parts are the most useful and important parts of a face. They tend to catch the maximum change among faces, specifically, the change that helps to differentiate one face from the other. This is how Eigen Faces face recognizer works. 


\section{SYSTEM ARCHITECHURE}

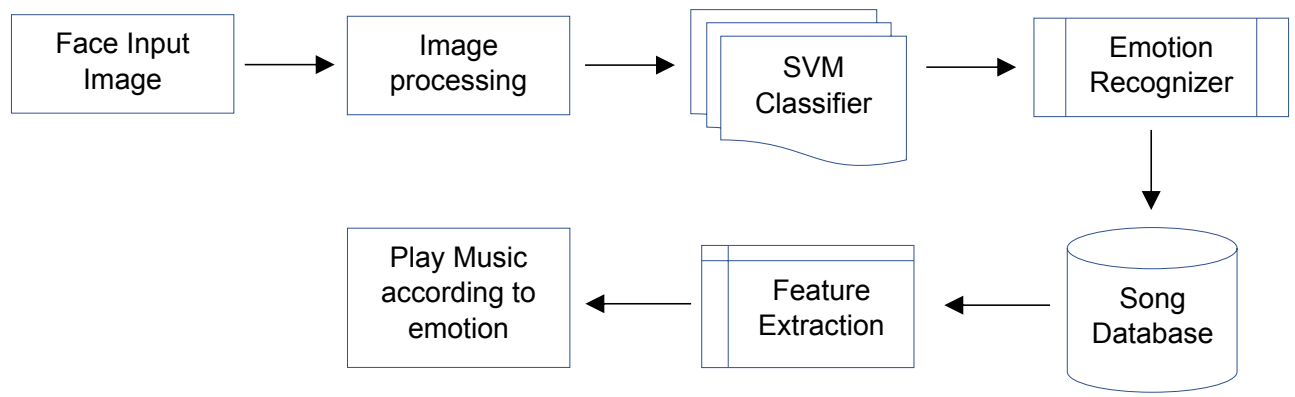

Graphic 1. Block Diagram.

The proposed framework is first prepared to distinguish a face from a static picture. When the information picture is perceived, the picture is handled. The picture isexposed to SVM classifiers for subtleties to perceive thefeeling displayed by the face. The subtleties recuperated from the image are utilized by the feeling classifier to discover feeling.

The song database and feature extraction module function simultaneously. The songs are disintegrated into several music pieces and the mood of the song is recognized. The songs are stored based on the mood detected. Once the emotion recognizer reports the mood, the songs pertaining to the mood are played by the music player.

\section{MODULE IDENTIFICATION}

Face Detection and Recognition: Facial expressions are powerful reflections of the emotional state of a person. In this section, we will discusshow images with human faces can be processed in order to detect the emotions presented in them. Various algorithms are used for face recognition. Here we are using the OpenCV to detect the face in the image. Eigenfaces algorithm is usedto recognize the face. The algorithms used for local feature extraction are Local Binary Patterns, Direct Cosines Transform, and Gabor Wavelets.

To depict progressively trademark highlights of thespecific chose face most noteworthy Eigenvalues of the Eigenvector will be picked as the ideal eigenface. Most noteworthy Eigenfaces with low Eigenvalues could be discarded since they coordinated just a little piece of trademark highlights of the countenances. 


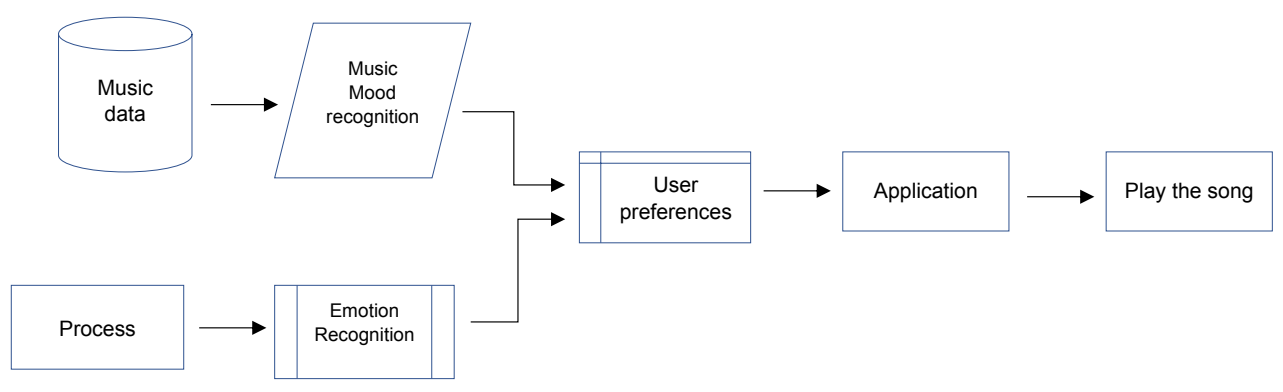

\section{Graphic 2. Module Explanation.}

Music Feature: Music can be recommended based on available information such as the album and artist. Another way of classifying the mood based on pitch and rhythm. Unfortunately, this will lead to predictable recommendations. For example, recommending songs basedon the artists that the user is known to enjoy is not particularly useful. With developing procedures, the utilization of Neural Networks has turned out to be progressively famous. We utilize an Artificial Neural Network (ANN) to arrange the melodies in individual classes. The dataset we utilized for preparing the model is Million Song Dataset given by Kaggle. The information comprises of two records: metadata document and triplet document. The metadat_file contains the title, song_id, artist_name, andrelease_by. Thetriplet_file contains user_id, song_id and listen time. 


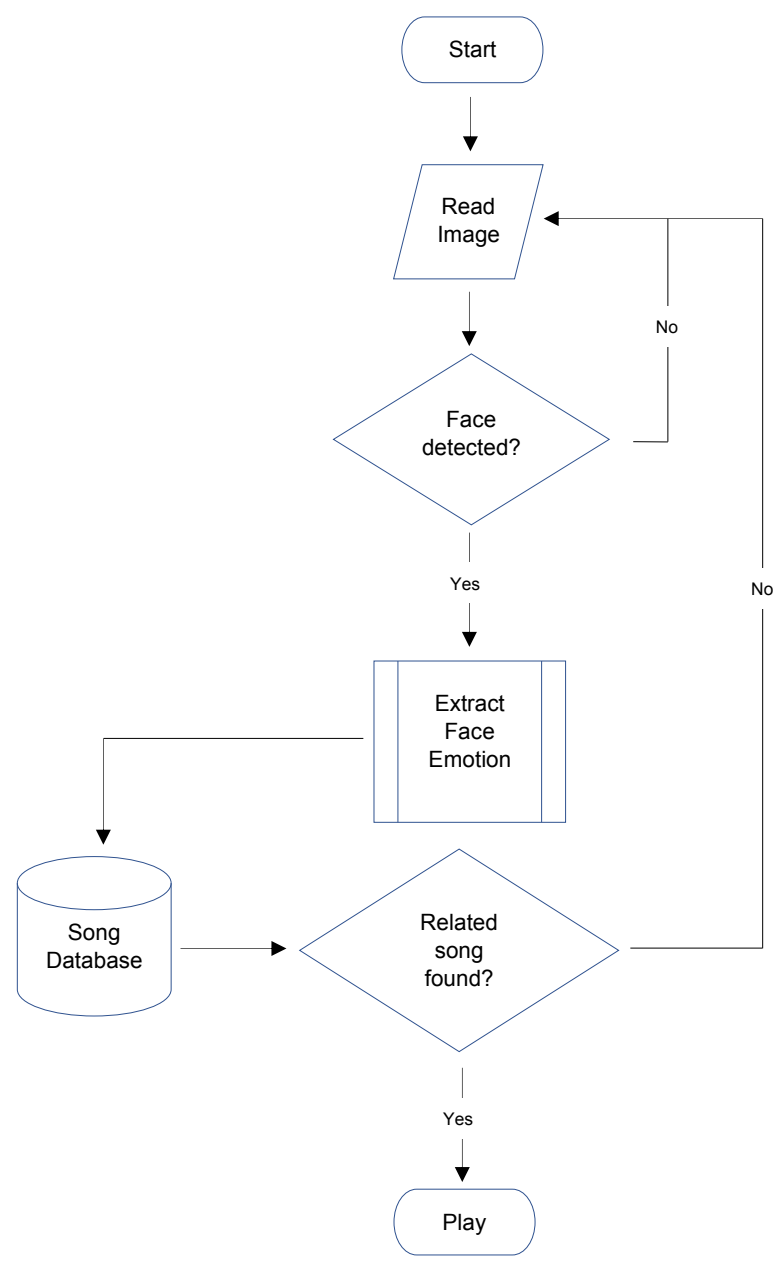

Graphic 3. Flow diagram of the proposed system.

\section{CONCLUSION AND FUTURE ENHANCEMENT}

A simple system is proposed here for the music recommendation using face emotion recognition. It suggests music by extracting different facial emotion of a person: Happy, anger, surprise, neutral. There is a degree for further upgrades and enhancements. Progressively effective approaches to incorporate different highlights and functionalities should, in any case, be investigated due to the lopsided nature of each element set. It is additionally seen that to improve the exactness of the arrangement framework the informational collection used to construct the grouping model could be expanded further. 


\section{REFERENCES}

Bhat, A. S., Amith, V. S., Prasad, N. S., \& Mohan, M. (2014). An Efficient Classification Algorithm For Music Mood Detection In Western and Hindi Music Using Audio Feature Extraction. 2014 Fifth International Conference on Signal and Image Processing, 359364. https://doi.org/10.1109/ICSIP.2014.63

Chew, L. W., Seng, K. P., Ang, L. M., Ramakonar, V., \& Gnanasegaran, A. (2011), Audio-Emotion Recognition System using Parallel Classifiers and Audio Feature Analyzer. In Proceedings of the 2011 Third International Conference on Computational Intelligence, Modelling \& Simulation (pp. 210-215), USA. IEEE. https://doi.org/10.1109/ CIMSim.2011.44

Deny, J., \& Sundararajan, M. (2014). Survey of Texture Analysis UsingHistogram in Image Processing. International Fournal of Applied Engineering Research, 9(26), 87378739. https://www.researchgate.net/publication/304091674_Survey_of_Texture_ Analysis_Using_Histogram_in_Image_Processing

Deny, J., \& Sundhararajan, M. (2015). Multi Modal Biometric Security for MANET Military Application-Face and Fingerprint. Fournal of Computational and Theoretical Nanoscience, 12(12), 5949-5953. https://www.researchgate.net/ publication/304380954_Multi_Modal_Biometric_Security_for_MANET_ Military_Application-Face_and_Fingerprint

Deny, J., Muthukumaran, E., Ramkumar, S., \& Kartheesawaran, S. (2018). Extraction Of Respiratory Signals And Motion Artifacts From PPG Signal Using Modified Multi Scale Principal Component Analysis. International fournal of Pure and Applied Mathematics, 119(12), 13719-13727. https://www.researchgate. net/publication/325465991_Extraction_Of_Respiratory_Signals_And_ Motion_Artifacts_From_PPG_Signal_Using_Modified_Multi_Scale_Principal_ Component_Analysis

Dureha, A. (2014). An Accurate Algorithm for Generating a Music laylist based on Facial Expressions. International Journal of Computer Applications, 100(9). https://pdfs. semanticscholar.org/312b/2566e315dd6e65bd42cfcbe4d919159de8a1.pdf 
Fan, X., Zhang, F., Wang, H., \& Lu, X. (2012). The System of Face Detection Based on OpenCV. In 24 th Chinese Control and Decision Conference (CCDC), Taiyuan, China. IEEE. https://doi.org/10.1109/CGDC.2012.6242980

Gilda, S., Zafar, H., Soni, G., \& Waghurdekar, K. (2017). Smart Music Player Integrating Facial Emotion Recognition and Music Mood Recommendation. In 2017 International Conference on Wireless Communications, Signal Processing and Networking (WiSPNET), Chennai, India. IEEE. https://doi.org/10.1109/WiSPNET.2017.8299738

Gossi, D., \& Gunes, M. H. (2016). Lyric-based music recommendation. In Cherifi H., Gonçalves B., Menezes R., Sinatra R. (eds.) Complex Networks VII. Studies in Computational Intelligence, vol. 644. Springer, Cham. https://doi.org/10.1007/978-3319-30569-1_23

Gupta, S. (2018). Facial emotion recognition in real-time and static images. In 2nd International Conference on Inventive Systems and Control (ICISC), Coimbatore, India. IEEE. https://doi.org/10.1109/ICISG.2018.8398861

Knyazev, B., Shvetsov, R., Efremova, N., \& Kuharenko, A. (2018). Leveraging large face recognition data foremotion classification. In 13th IEEE International Conference on Automatic Face $\mathcal{E}$ Gesture Recognition (FG 2018), Xi'an, China. IEEE. https://doi. org/10.1109/FG.2018.00109

Levi, G., \& Hassner, T. (201 1). Emotion Recognition in the Wild via Convolutional Neural Networks and Mapped Binary Patterns. In Proceedings ACM International Conference on Multimodal Interaction (ICMI), Seattle. https://doi.org/10.1 145/2818346.2830587

Muthukumaran, E., Deny, J., Perumal, B., Suseendran, G., \& Akila, D. (2015). A narrative Non-Invasive Diagnostic loom Based by the side of correlation of Nasal set Rhythm in addition to customary Three Radial Pulses Measurement. Fournal of Physics: Conference Series, 1228(1). https://iopscience.iop.org/ article/10.1088/1742-6596/1228/1/012075 
Sankaranarayanan, S., \& Deny, J. (2013). Securable Image Compression Using SPIHT Algorithm. International journal of Electronics and Communication Engineering \& Technology, $4(4)$, 96-100. https://fdocuments.in/document/securable-image-compressionusing-spiht-algorithm.html

Tzanetakisand, G., \& Cook, P. (2002). Musical Genre Classification of Audio Signals. IEEE Transactions on Speech and Audio Processing, 10(5). https://doi.org/10.1109/ TSA.2002.800560

Youssif, A. A. A., \& Wesam, A. A. A. (2011). Automatic Facial Expression Recognition System based on Geometric and Appearance Features. Computer and Information Science, 4(2), 115-124. https://doi.org/10.5539/cis.v4n2p115

Zeng, Z., Pantic, M., Roisman, G. I., \& Huang, T. S. (2009). A survey of affect recognition methods: Audio, visual, and spontaneousexpressions. IEEE Transactions on Pattern Analysis and Machine Intelligence, 31(1). https://doi.org/10.1109/TPAMI.2008.52 